\title{
Development and Evaluation of Temperature Mediated In-Situ Mucoadhesive Gel of Lamotrigine for Intranasal Administration
}

\section{IJCRR}

Section: Healthcare

ISI Impact Factor

(2020-21): 1.899

IC Value (2020): 91.47

$\operatorname{SJIF}(2020)=7.893$

(c) (7) (9)

Copyright@IJCRR

\section{Rathi Tejas, Khetade Roshan, Dhande Payal, Das Renuka, Umekar Milind, Taksande Jayshree*}

Department of Pharmaceutics. Smt. Kishoritai Bhoyar College of Pharmacy, Kamptee Rashtrasant Tukadoji Maharaja University, Nagpur-441002., India.

\section{ABSTRACT}

Introduction: Epilepsy a neurodegenerative disease causes spontaneous and repetitive seizures. Treatment requires instantaneous pharmacotherapy to prevent further the condition of status epilepticus.

Aim: The present study was aimed at developing temperature-sensitive in situ gel for intranasal administration of lamotrigine, an anticonvulsant drug used to treat generalised and partial seizure.

Methodology: Pluronic 127, chitosan and $\beta$-glycerophosphate were used for the preparation of gel in varying concentrations by cold method. These systems were characterized for physical properties such as $\mathrm{pH}$, gelling temperature, gelling time, drug content, in vitro drug diffusion studies, ex-vivo drug permeation and histopathological studies. The drug polymer compatibility was studied using Fourier transform infrared spectroscopy.

Results: All the prepared formulations gelled immediately below $25 \mathrm{sec}$ at the nasal $\mathrm{pH}$ and temperature. Addition of chitosan with Pluronic 127 increases the mucoadhesion and contact time of formulation in nasal cavity. The result of in-vitro drug release study revealed $93 \%$ of Lamotrigine and $75.33 \%$ of drug release in ex-vivo permeability study from the optimized formulation in about $210 \mathrm{~min}$. Result of histopathological studies suggests the suitability of prepared formulation for intranasal administration.

Conclusion: In-situ intranasal gel of lamotrigine prepared by using Pluronic 127 and chitosan demonstrated gelation at body temperature and exhibited satisfactory release of drug from its dosage form. It can be concluded that the prepared formulation has potential for the intranasal administration of lamotrigine in the treatment of epilepsy.

Key Words: In-situ gel, Mucoadhesive, Intranasal administration, Lamotrigine, Temperature-sensitive, Epilepsy

\section{INTRODUCTION}

Epilepsy is a neurological disorder of brain characterized by sudden recurrent episodes of sensory disturbances causing seizures. Increased and unusual nerve cell activity in the cortex of the brain results in epileptic seizures. ${ }^{1}$ Some occur as a result of tumour of brain, brain injury and brain defect stroke. ${ }^{2}$ About 3-10 per 1000 people have epilepsy worldwide. ${ }^{3}$ Selection of AED for a patient depends upon the affected individual's condition and side effects of drug. ${ }^{4}$ The nasal mucosa is highly vascularised responsible for the transfer of drug through the mucus layer leads to the absorption. ${ }^{5}$ A drug molecule can be transferred quickly across the single epithelial cell layer directly to the systemic blood cir- culation without first-pass hepatic and intestinal metabolism. Nowadays nasal delivery has been focused as an alternative route of drug administration. The advantages of the nasal administration include rapid absorption, higher bioavailability, fast onset of therapeutic action, avoidance of presystemic metabolism, non-invasive administration and improved patient compliance. ${ }^{6}$ The poor bioavailability and therapeutic response exhibited by the conventional nasal solution due to short residence time is the basic problem of highly efficient nasal route. Mucoadhesive in situ gelling formulation is one of the approaches to enhance the residence time of the drug in the nasal cavity leading to improved drug absorption and its therapeutic effect with rapid onset of action. Mucoadhesive system aims in targeting and localization of the dosage

\section{Corresponding Author:}

Dr. Jayshree Taksande, Associate Professor, Smt. Kishoritai Bhoyar College of Pharmacy, Kamptee, Dist-Nagpur-441002, Maharashtra, India. Email: jayabtaksande@gmail.com

ISSN: $2231-2196$ (Print)

Received: 16.09 .2021
ISSN: 0975-5241 (Online)

Revised: 09.10 .2021
Accepted: 30.10 .2021 
forms and provide an intimate contact between dosage form and absorptive mucosa resulting in high retention time and greater absorption of drug. ${ }^{7,8}$

In situ liquid polymeric formulations once administered undergo in situ gelation. Various approaches used for in situ gelling system include physiological stimuli, osmotic stimuli, chemical stimuli, pH-triggered system, and temperaturedependent system. ${ }^{9}$ Temperature is most widely used stimulus in environmentally responsive polymer system. In these systems, gelling of the solution is triggered by change in temperature. These hydrogels are liquid at room temperature $\left(20-25^{\circ} \mathrm{C}\right)$ and undergo gelation with increase in temperature when in contact with body fluid $\left(35-37^{\circ} \mathrm{C}\right) .{ }^{10}$ The polymers which show temperature-induced gelation include chitosan, pluronics, tetronics, xyloglucans, and hydroxyl propyl methyl cellulose. ${ }^{11}$

Lamotrigine, an antiepileptic drug regularly used to treat partial seizures and tonic-clonic convulsions. ${ }^{12}$ Lamotrigine has relatively few side-effects and does not require therapeutic drug monitoring. Lamotrigine is available as tablet and intravenous formulation. In view of the potential pharmacological application of lamotrigine in the treatment of epilepsy, the present work was designed to formulate its mucoadhesive temperature-sensitive in situ gel for treating acute and emergency epileptic condition.

\section{MATERIAL AND METHODS}

\section{Materials}

Lamotrigine was obtained as a generous gift sample from Lupin pharma, Pune, India. Chitosan, pluronic127, $\beta$-glycerophosphate, benzalkonium chloride were purchased from Sigma Drug Laboratory Pvt. Ltd. All the chemicals used in experiments were of analytical grade.

\section{Methods}

\section{Formulation of In-Situ Gel}

Table 1 depicts the concentration of lamotrigine, chitosan and pluronic F 127 used for the preparation of various batches of formulations. The concentration of Pluronic F127 was selected based on initial studies so as to obtain gel at minimum possible concentration below $34^{\circ} \mathrm{C}$ and $\mathrm{pH}$ of 4.8 . Chitosan was used as mucoadhesive polymer to increase the gel strength. Benzalkonium chloride was added as preservative and $\beta$-glycerophosphate was used as antioxidant to protect the formulation from microorganisms and oxidation. Distilled water was used as vehicle.

The gel was prepared by cold method. Initially $2 \% \mathrm{w} / \mathrm{v}$ chitosan solution was prepared in $0.1 \mathrm{M} \mathrm{HCl}$ in ice bath, to it $10 \% \mathrm{w} / \mathrm{v}$ of glycerophosphate solution was added dropwise with continues stirring. Separately, pluronic F127 solutions of different concentration was prepared and kept these solutions in ice bath for removal of foam. Further chitosan solution was added to pluronic F127solution with continues stirring. ${ }^{13,14}$

\section{Calibration curve of Lamotrigine}

A solution of $100 \mu \mathrm{g} / \mathrm{ml}$ of lamotrigine was scanned in the range of 400 to $200 \mathrm{~nm}$ for the determination of $\lambda$ max. Calibration curve of lamotrigine was plotted for the concentrations $2-20 \mu \mathrm{g} / \mathrm{ml}$ in phosphate buffer $\mathrm{pH}$ 6.6.

\section{Physicochemical Characterization}

Formulated in-situ intranasal gel was critically analysed for physicochemical properties such as clarity, $\mathrm{pH}$, gelation time and gelation temperature. Clarity is one of the most important characteristics features of gel formulation. All the prepared gel formulations were evaluated for clarity by visual observation against black and white background. ${ }^{15} \mathrm{pH}$ of each formulation was determined by using digital $\mathrm{pH}$ meter which was previously calibrated using buffer of $\mathrm{pH} 4$ and $\mathrm{pH}$ 7. The $\mathrm{pH}$ values were recorded immediately after preparation of gel.

Gelation temperature and gelation time of various gel preparations were determined by taking formulation equivalent to $10 \mathrm{mg}$ of drug in a test tube and immersed in a water bath. Temperature of water bath was increased slowly and left to equilibrate for $5 \mathrm{~min}$ at each new setting. The sample was then examined for gelation, which was said to have occurred when the meniscus would no longer move upon tilting through $90^{\circ} \mathrm{C}$. Gelation time was measured as the time at which gel does not flow at $37^{\circ} \mathrm{C}$ on immersing the solutions in a thermostatic water bath.

\section{Fourier Transform Infrared Spectroscopy (FTIR)}

FTIR spectra of the pure drug, physical mixture of drug with polymers were obtained using FTIR (Thermo Nicolet, Avatar 370). The samples were prepared as potassium bromide $(\mathrm{KBr})$ disks on an FTIR spectrophotometer (2 $\mathrm{mg}$ sample in $200 \mathrm{mg} \mathrm{KBr}$ ratio) and scanned in a range $400-4000 / \mathrm{cm}$ as described earlier. ${ }^{16}$ FTIR spectra were taken to investigate compatibility between the drug and the polymer.

\section{Differential Scanning Calorimeter (DSC)}

Differential scanning calorimetric (DSC) was used to evaluate the thermal behaviour of pure drug and physical mixture of the drug and excipients. The thermal profile of mentioned samples was recorded on DSC (Mettler Toledo DSC 822e). The thermograms were obtained by heating the microspheres at rate of $10^{\circ} \mathrm{C} / \mathrm{min}$ from $30^{\circ} \mathrm{C}$ to $300^{\circ} \mathrm{C}$ using nitrogen purge of $50 \mathrm{ml} / \mathrm{min}^{17}$ 


\section{X-ray Diffraction Studies (XRD)}

$\mathrm{X}$ - ray diffractogram analysis provides information about crystalline and amorphous nature of the substances. Diffractograms were recorded for the pure drug and formulation on X-ray diffractometer (Brucker AXS D8) to evaluate its crystallinities. Diffractograms were scanned in the range from $3^{\circ} \mathrm{C}$ to $80^{\circ} \mathrm{C}(2 \theta)$ with resolution of $0.02^{\circ} \mathrm{C}$ and scanning speed of $2.0^{\circ} \mathrm{C} / \mathrm{min}$. An accelerating voltage of $40 \mathrm{kV}$ was applied at the current intensity of $35 \mathrm{~mA}$. The XRD pattern of in situ gel formulation was compared with that of the pure drug. ${ }^{4}$

\section{Drug Content}

Drug content of prepared formulations was determined by dissolving $1 \mathrm{ml}$ gel formulation into $50 \mathrm{ml}$ of $\mathrm{pH} 6.6$ phosphate buffer solution. The resultant solution was analysed for the drug content by ultraviolet (UV)-visible spectrophotometer at $306 \mathrm{~nm}$ (Shimadzu 7800, Tokyo Japan). pH 6.6 phosphate buffer was used as blank. The data were collected by repeating the procedure in triplicate.

\section{In vitro drug diffusion studies}

The in vitro drug diffusion study of gel formulations was carried out using Franz diffusion cell across dialysis membrane (Av diameter $21.5 \mathrm{~mm}$, Av flat width $32.34 \mathrm{~mm}$ ) as diffusion barrier. The membrane was equilibrated overnight with $\mathrm{pH}$ 6.6 phosphate buffer before application of gel onto the donor compartment. The receptor compartment was filled with phosphate buffer solution $(\mathrm{pH}$ 6.6) that was within the $\mathrm{pH}$ range in the nasal cavity. The donor compartment was placed in such a way that it just touched the diffusion medium in the receptor compartment. The temperature was maintained at $37^{\circ} \mathrm{C} \pm 1{ }^{\circ} \mathrm{C}$ using circulating water bath. $300 \mu 1$ of sample was withdrawn at predetermined time points from the receptor compartment, replaced with the same amount of fresh pre-warmed buffer solution and analysed for lamotrigine using UV-visible spectrophotometer at $306 \mathrm{~nm} .{ }^{18}$

\section{Ex Vivo Permeation Studies}

For the purpose of this study, fresh nasal tissue of goat was obtained from the local slaughterhouse. The nasal mucosa was separated from septum and the connective tissue as well as most of the adhering cartilaginous tissue was carefully removed with forceps and scissors without damaging or scratching the nasal mucosa. The specimen was individually placed on Franz diffusion cell and clamped between the donor and receptor compartments. $18 \mathrm{ml}$ of $\mathrm{pH} 6.6$ phosphate buffer was maintained at $37^{\circ}$ was placed in the receptor compartment. Formulation equivalent to $10 \mathrm{mg}$ of lamotrigine was placed in the donor compartment. Sampling was done in a similar manner as for in vitro release studies. The amount of lamotrigine diffused into the receptor phase from the formulations was determined by UV spectrophotometry at 306 $\mathrm{nm}$. The method was found to be sensitive enough for detecting the amount of drug permeated. ${ }^{18}$

\section{Ex vivo biocompatibility study}

The histopathological studies were accomplished to confirm the biocompatibility of lamotrigine intranasal in-situ gel formulation with goat nasal mucosa. The freshly excised nasal mucosa of goat was collected and cleaned with saline solution as mentioned in vitro drug diffusion study. After application of predefined amounts of lamotrigine in-situ gel (1 $\mathrm{mg}$ ), mucosal tissues were fixed in $10 \%$ formalin solution and implanted in paraffin. Paraffin sections $(7.5 \mu \mathrm{m})$ were stained with Hematoxylin Eosin and observed under digital Motic microscope (Model no B1-223SP). The untreated mucosa incubated with phosphate buffer solution ( $\mathrm{pH} \mathrm{6.6)} \mathrm{was}$ used as a control. ${ }^{19}$

\section{Statistical analysis}

All the results are reported as mean \pm standard deviation (SD) or mean \pm standard error mean (SEM)

\section{RESULT AND DISCUSSION}

In-situ temperature-sensitive intranasal mucoadhesive gel of lamotrigine was prepared by cold method. Initially various concentrations of Pluronic F127 were used to obtain formulation that forms gel at temperature near $34^{\circ} \mathrm{C}$ and $\mathrm{pH}$ of 4.8. Formulations showed adequate gelation in proper time at concentration of $32 \% \mathrm{w} / \mathrm{v}$ and $34 \% \mathrm{w} / \mathrm{v}$. Chitosan concentration was also varied to obtain gel having suitable viscosity. Based on these studies' concentration of chitosan and Pluronic F127 was selected for the further formulations. The purity of lamotrigine was checked by determining the melting point of it by capillary method which was found to be $216^{\circ} \mathrm{C}-218^{\circ} \mathrm{C}$. The drug exhibited the $\lambda$ max at $306 \mathrm{~nm}$ and showed reproducibility. Calibration curve of lamotrigine obeyed Beers-Lambert's law in the concentration range 2-20 $\mu \mathrm{g} / \mathrm{ml}$ (Figure 1).

\section{Physicochemical Characterization}

All the formulations showed the $\mathrm{pH}$ in the range of 4.87 \pm 0.16 to $5.37 \pm 0.08$, which is suitable according to the nasal $\mathrm{pH}$. All the formulations were clear in appearance in sol form. The gelling temperature of the prepared formulations were in the range $32.23 \pm 1.87$ to $35.30 \pm 1.22{ }^{\circ} \mathrm{C}$. In the present study, all the formulations showed gelation temperature within the acceptable range. The gelling temperature suitable for temperature-sensitive in-situ nasal gel ranges between $30-36^{\circ} \mathrm{C}$. The gelation point refers to the temperature when the meniscus of the formulation would no longer move upon slanting the test tubes at $90^{\circ}$, with gradual increase in the temperature. The gelling time of all the formulations was found in the range of $15.06 \pm 0.90$ to $25.33 \pm 4.48 \mathrm{sec}$. All the 
formulations exhibited satisfactory gelation time for intranasal administration for lamotrigine (Table 1).

\section{Fourier Transform Infrared Spectroscopy}

FTIR spectra of lamotrigine showed characteristic absorbance at $3448 \mathrm{~cm}-1$ specifying $\mathrm{NH}$ stretching of an amino group (Aromatic), $3209 \mathrm{~cm}-1$ shows aromaticity (aromatic $\mathrm{CH}$ stretching), $1620 \mathrm{~cm}-1,1486 \mathrm{~cm}-1$ indicates $\mathrm{C}=\mathrm{C}$ ring stretching and $962 \mathrm{~cm}-1$. In FTIR spectra of physical mixture characteristics peak of both lamotrigine and excipients were observed. It suggests no significant interaction between drug and excipient. However, the characteristic absorbance peaks of lamotrigine were found to be shifted to the lower values and decreased in intensity also, suggesting the incorporation of lamotrigine within in-situ gel prepared with Pluronic F127 and chitosan as carriers (Figure 2).

\section{X-ray Diffraction Studies}

The X-ray diffractogram of lamotrigine shows a sharp and intense peak indicating the crystalline nature of the drug in pure form. The X-ray diffractogram of the physical mixture was observed to determine if there is a loss or modification of the pure drug's crystal structure. Figure 3 indicates that there is virtually no difference in crystallinity between physical mixture of drug with excipients and pure lamotrigine proposed compatibility between the lamotrigine and the excipients.

\section{Drug content}

Drug content of the formulations was found to increase with the increase in concentration of pluronic F 127 and chitosan. The drug content of the formulations was ranging from 48.35 $\pm 3.55 \%$ to $69.12 \pm 2.77 \%$, indicating maximum entrapment of drug in the formulation (Table 2). The maximum amount of drug content $69.12 \pm 2.77 \%$ was obtained for in-situ gel formulation with $34 \%$ of Pluronic F127 and $8 \%$ of chitosan concentration.

\section{In vitro drug diffusion study}

The release of lamotrigine from the in situ intranasal formulations was determined using Franz diffusion cell. The receptor compartment was filled with phosphate buffer $\mathrm{pH} 6.6$ as the dissolution medium. In vitro drug diffusion study revealed that the release rate dependent on Pluronic F127 and chitosan concentration. Higher the pluronic F127 concentration, greater the rate of drug release was observed from the formulations. However, formulations with greater chitosan concentration decreased the rate of drug release might be due to increased viscosity of the gel formulation. With the concentration of chitosan $2 \%$ and $4 \%$, showed the greater release of lamotrigine. Bioadhesive property of chitosan is responsible to retain the formulation at the administration site helps to increase the retention time, also increases the transmembrane permeability of drug and thereby exhibit bet- ter absorption of the drug. The formulation F6 containing $34 \% \mathrm{w} / \mathrm{v}$ of Pluronic F127 and 4\% w/v of chitosan concentration showed $93 \%$ of drug release in 270 min (Figure 4). The same formulation was selected further for the ex- vitro permeation study and histopathological study.

\section{Ex-vivo permeation study}

The ex-vivo permeation study of formulations F6. Ex-vivo permeation study was performed for the significant batch using goat nasal mucosa. The percent drug permeated after 210 min was found to be $85.18 \pm 2.28 \%$ (Figure 5) from nasal gel formulation showing significant release of drug from the formulation.

\section{Ex vivo biocompatibility study}

The epithelium layer was intact and there were no alterations in basal membrane and superficial part of sub mucosa as compared with PBS treated mucosa (Figure 6). Thus, insitu gel formulations seem to be safe with respect to nasal administration.

\section{CONCLUSION}

In this study we have formulated intranasal mucoadhesive in-situ temperature-sensitive gel of lamotrigine. Physicochemical characterization exhibited satisfactory gelation of formulation in $25 \mathrm{sec}$. In-vitro and ex-vivo drug permeation studies have shown that in situ gels act as potential drug delivery system for lamotrigine. Histopathological study revealed the safe intranasal administration of in-situ gel formulation. Present study highlighted the importance of intranasal administration of lamotrigine as mucoadhesive in-situ temperature-sensitive gel for emergency conditions of epileptic seizures. However, in vivo studies are required to be carried out to confirm the potential of these formulations.

\section{ACKNOWLEDGEMENT}

The authors are thankful to the Smt. Kishoritai Bhoyar College of Pharmacy, Kamptee management for providing research facilities and support for carrying out this study.

\section{Funding Information}

There is no financial support received any government or non-government funding agency.

\section{Conflict of Interest}

The author stated no conflict of interest for the publication of this research article.

Rathi Tejas- Data collection, methodology, formal analysis and investigation.

Khetade Roshan- Data collection, methodology, formal analysis and investigation. 
Dhande Payal- Data collection, methodology, formal analysis and investigation.

Taksande Jayshree- Supervision, validation, writing original draft and editing.

Das Renuka- Supervision, validation, writing original draft and editing.

Umekar Milind- Supervision, validation, writing original draft and editing.

\section{REFERENCES}

1. Tan L, Yu JT, Guan HS. Intranasal anticonvulsive treatment: prospective management of intractable epilepsy? Med Hypotheses 2008; 71: 542-5.

2. Kumar S, Mohanty BB, Agrawal D, Nayak P, Patnaik S, Patnaik J, Mahapatra SK. Antiepileptics and Pregnancy: A Review. Int. J. curr Res Rev 2012;4(21):132-43.

3. Taksande V, Burbare N, Chaure K, Deogade N, Deshmukh S, Dhole J. To Assess the Effectiveness of Planned Teaching on the Knowledge Regarding Epilepsy in Children Among the Anganwadi Workers. Int. J. curr Res Rev 2000;12(24):151-54.

4. Paul A, Fathima KM, Nair SC. Intra Nasal In situ Gelling System of Lamotrigine Using Ion Activated Mucoadhesive Polymer. The open medicinal chem J 2017; 11: 222-44.

5. Chitra Karthikeyini S, Kavitha K. Nasal Drug Delivery-A Pre Hospital Therapy in Status Epilepsy-Review. IOSR J. Pharm. 2016; 11(3): 39-46.

6. Soane RJ, Hinchcliffe M, Davis SS, Illum L. Clearance characteristics of chitosan based formulation in the sheep nasal cavity. Int J Pharm 2001; 217: 183-191.

7. Kukudkar P, Rahate S, Trivedi R, Umekar M, Taksande J. Intranasal Topiramate Polymeric Nanoparticles for Epilepsy: In Vitro And Ex-Vivo Investigation. Int J App Pharm 2020; 12(5): 258-64.

8. Agrawal V.A, Rajurkar R.M, Mahle A.M, Thonte S.S. Sustained Intranasal Drug Delivary System For The Treatment of Gastroparesis. International Journal of Current Research and Review 2011; 03 (02): 4-12.
9. Caramella CM, Rossi S, Ferrari F, Bonferoni MC, Sandri G. Mucoadhesive and thermogelling systems for vaginal drug delivery. Advanced Drug Delivery Reviews 2015: 92, 39-52.

10. Karavasili C, Fatouros DG. Smart materials: in situ gel-forming systems for nasal delivery. Drug Discovery Today 2016; 21(1): 157-66.

11. Jagdale S, Shewale N, Kuchekar BS. Optimization of Thermoreversible In Situ Nasal Gel of Timolol Maleate. Scientifica (Cairo). 2016; 2016: 6401267.

12. Schiller Y, Krivoy N. Safety and efficacy of lamotrigine in older adults with epilepsy and co-morbid depressive symptoms. Clin Med Ther 2009; 1: 825-33.

13. Nazar H, Fatouros DG, Van der Merwe SM, Bouropoulos N, Avgouropoulos G, Tsibouklis J, Roldo M. Thermosensitive hydrogels for nasal drug delivery: The formulation and characterisation of systems based on N-trimethyl chitosan chloride. European J. P'ceutics \& Biopharmaceutics 2011; 77: 225-32.

14. Altuntas E, Yener G. Formulation and Evaluation of Thermoreversible In Situ Nasal Gels Containing Mometasone Furoate for Allergic Rhinitis. AAPS Pharm Sci Tech 2017;18(7): 2673-82.

15. Saudagar RB, Deore SB, Gondkar SB. Formulation development and evaluation of in-situ nasal gel of lisinopril dehydrate. Sch Acad J Pharm 2016; 5(7): 277-83.

16. Gangurde H, Chavan N, Mundada A, Derle D, Tamizharasi S. Biodegradable chitosan-based ambroxol hydrochloride microspheres: Effect of cross-linking agents. J Young Pharm 2011; 3: 9-14.

17. Patil PR, Salve VK, Thorat RU, Shahi SR. Formulation and evaluation of ion-sensitive in-situ gel of zolmitriptan. Int J Pharmacy and Pharm Sci 2014; 7(1): 478-86.

18. Naik A, Nair H. Formulation and evaluation of thermosensitive bio gels for nose to brain delivery of doxepin. Hindawi Pub Co Scientifica BioMed Research International 2014; Volume 2014, Article ID 847547, 10 pages.

19. Pardeshi CV, Belgamwar VS, Tekade AR, Surana SJ. Novel surface modified polymer-lipid hybrid nanoparticles as intranasal carriers for ropinirole hydrochloride: In vitro, ex vivo and in vivo pharmacodynamic evaluation. J Mater Sci Mater Med 2013; 24: 2101-15.

\section{Table 1: Composition of different batches of lamotrigine loaded in-situ intranasal gel}

\begin{tabular}{lcccccccc} 
Ingredients & $\mathbf{F 1}$ & $\mathbf{F}_{2}$ & $\mathbf{F}_{3}$ & $\mathbf{F}_{4}$ & $\mathbf{F}_{5}$ & $\mathbf{F 6}$ & $\mathbf{F}_{7}$ & F8 \\
Pluronic F127 $(\% \mathrm{w} / \mathrm{v})$ & 32 & 32 & 32 & 32 & 34 & 34 & 34 & 34 \\
Chitosan $(\% \mathrm{w} / \mathrm{v})$ & 2 & 4 & 6 & 8 & 2 & 4 & 6 & 8 \\
Lamotrigine $(\mathrm{mg})$ & 100 & 100 & 100 & 100 & 100 & 100 & 100 & 100 \\
\hline
\end{tabular}

Table 2: pH, Gelling Temperature, Gelling Time and Drug Content of various in-situ gel formulations.

\begin{tabular}{lcccc} 
Formulations & $\mathbf{p H}$ & Gelling Temperature $(\mathrm{C})$ & Gelling Time(Sec) & Drug Content(\%) \\
F1 & $4.87 \pm 0.16$ & $34.06 \pm 1.00$ & $16.43 \pm 3.73$ & $48.26 \pm 13.55$ \\
F2 & $5.24 \pm 0.04$ & $35.30 \pm 1.22$ & $19.83 \pm 5.22$ & $50.35 \pm 2.98$ \\
F3 & $5.28 \pm 0.03$ & $35.06 \pm 0.50$ & $20.63 \pm 2.50$ & $52.97 \pm 20.45$ \\
F4 & $5.22 \pm 0.04$ & $34.00 \pm 1.24$ & $18.00 \pm 1.99$ & $56.39 \pm 32.34$ \\
F5 & $5.37 \pm 0.08$ & $32.23 \pm 1.87$ & $16.87 \pm 2.70$ & $65.12 \pm 18.77$ \\
F6 & $5.13 \pm 0.07$ & $33.93 \pm 0.41$ & $17.26 \pm 0.64$ & $67.67 \pm 1.24$ \\
F7 & $5.06 \pm 0.03$ & $31.76 \pm 1.16$ & $15.06 \pm 0.90$ & $68.67 \pm 12.73$ \\
F8 & $5.04 \pm 0.03$ & $33.26 \pm 0.30$ & $17.43 \pm 4.36$ & $69.22 \pm 30.18$ \\
\hline
\end{tabular}

Results are shown as mean \pm SD $(n=3)$ 


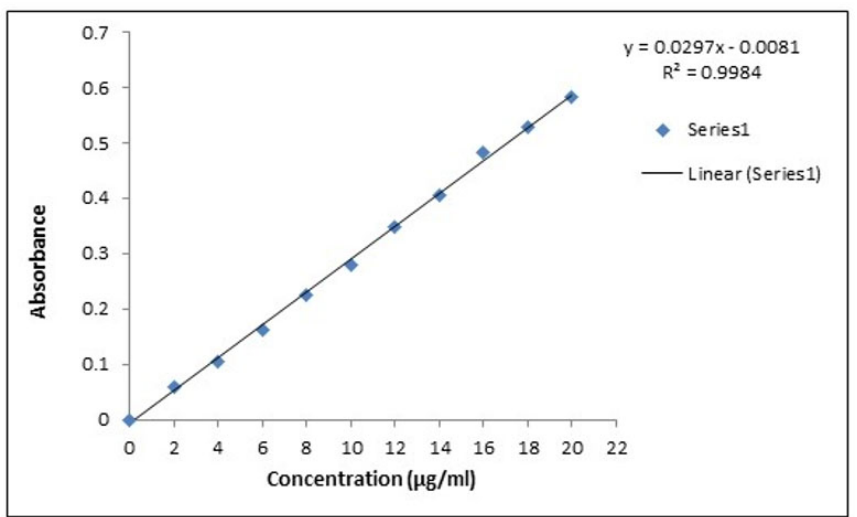

Figure 1: Calibration curve of lamotrigine in $\mathrm{pH} 6.6$ phosphate buffer.

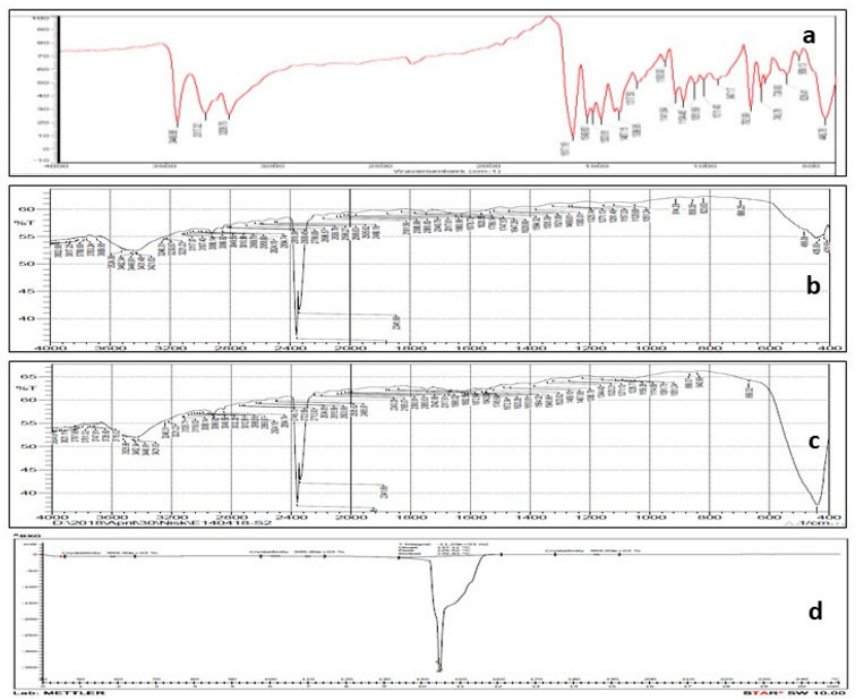

Figure 2: FTIR spectra: a- Lamotrigine, b- Physical mixture of lamotrigine and PF127, c- Physical mixture of lamotrigine and chitosan, d- In-situ gel Formulation.

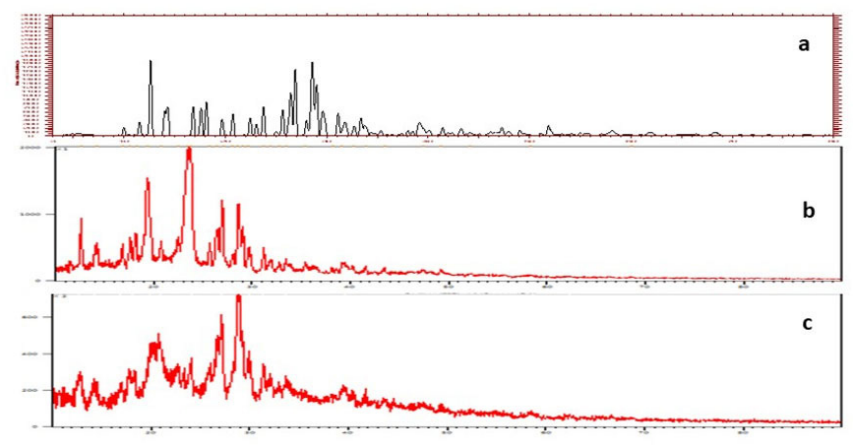

Figure 3: XRD: a- Lamotrigine, b- Physical mixture of lamotrigine and PF127, c- Physical mixture of lamotrigine and chitosan.

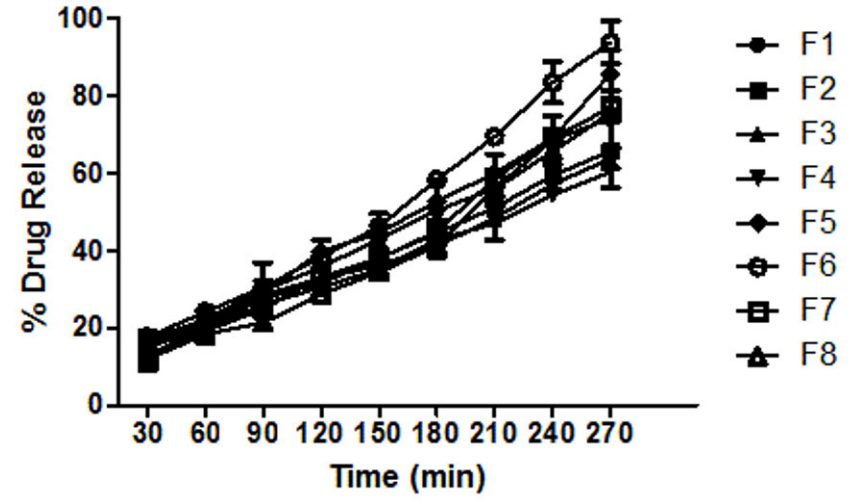

Figure 4: In-vitro drug diffusion study of in situ nasal gel formulations.

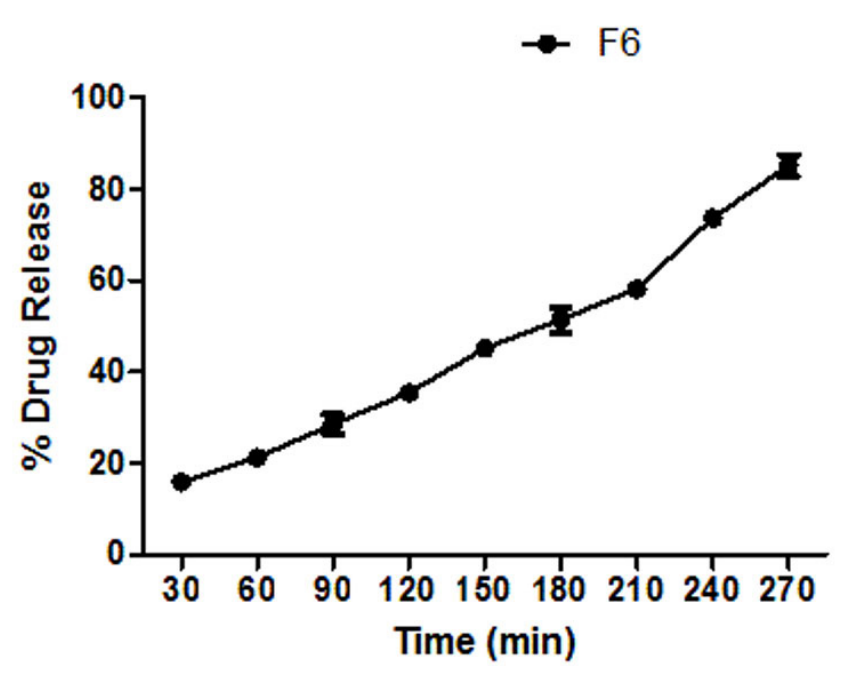

Figure 5: Ex-vivo permeation of in-situ gel through nasal mucosa.
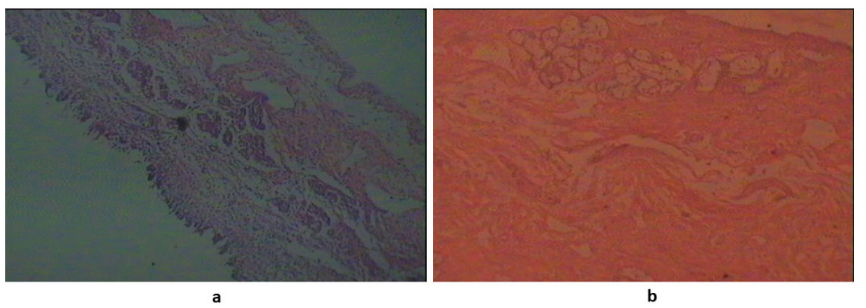

Figure 6: Light photomicrograph of goat nasal mucosa, A) untreated control mucosa and (B) In-situ lamotrigine gel - treated mucosa. 